\title{
Solar Nantenna Electromagnetic Collectors
}

\section{$2^{\text {nd }}$ International Conference on Energy Sustainability}

\author{
Dale K. Kotter
}

Steven D. Novack

W. Dennis Slafer

Patrick Pinhero

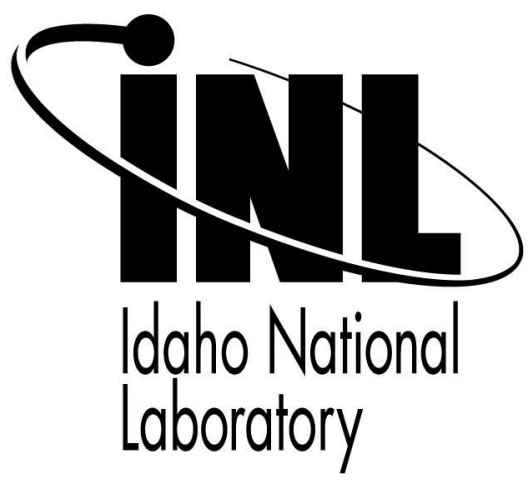

\section{August 2008}

This is a preprint of a paper intended for publication in a journal or proceedings. Since changes may be made before publication, this preprint should not be cited or reproduced without permission of the author. This document was prepared as an account of work sponsored by an agency of the United States Government. Neither the United States Government nor any agency thereof, or any of their employees, makes any warranty, expressed or implied, or assumes any legal liability or responsibility for any third party's use, or the results of such use, of any information, apparatus, product or process disclosed in this report, or represents that its use by such third party would not infringe privately owned rights. The views expressed in this paper are not necessarily those of the United States Government or the sponsoring agency. 


\section{ES 2008-54016}

\section{SOLAR NANTENNA ELECTROMAGNETIC COLLECTORS}

\author{
Dale K. Kotter \\ Idaho National Laboratory \\ W. Dennis Slafer \\ MicroContinuum, Inc.
}

\author{
Steven D. Novack \\ Idaho National Laboratory \\ Patrick Pinhero \\ University of Missouri
}

\begin{abstract}
This research explores a new efficient approach for producing electricity from the abundant energy of the sun. A nantenna electromagnetic collector (NEC) has been designed, prototyped, and tested. Proof of concept has been validated. The NEC devices target mid-infrared wavelengths, where conventional photovoltaic (PV) solar cells are inefficient and where there is an abundance of solar energy. The initial concept of designing NEC was based on scaling of radio frequency antenna theory. This approach has proven unsuccessful by many due to not fully understanding and accounting for the optical behavior of materials in the $\mathrm{THz}$ region. Also, until recent years the nanofabrication methods were not available to fabricate the optical antenna elements. We have addressed and overcome both technology barriers.

Several factors were critical in successful implementation of NEC including: 1) frequency-dependent modeling of antenna elements; 2) selection of materials with proper $\mathrm{THz}$ properties; and 3) novel manufacturing methods that enable economical large-scale manufacturing. The work represents an important step toward the ultimate realization of a low-cost device that will collect, as well as convert this radiation into electricity, which will lead to a wide spectrum, high conversion efficiency, and low-cost solution to complement conventional PVs.
\end{abstract}

Keywords- Nantenna, Frequency Selective Surfaces, nanoscale modeling, nano-fabrication, nantennas

\section{INTRODUCTION}

Full spectrum incident and reflective (readmitted) electromagnetic (EM) radiation originating from the sun provides a constant energy source to the earth. Approximately $30 \%$ of this energy is reflected back to space from the atmosphere, $19 \%$ is absorbed by atmospheric gases and reradiated to the earth's surface in the mid-IR range (7-14 um), and $51 \%$ is absorbed by the surface or organic life and reradiated at around $10 \mathrm{um}$ [1]. The energy reaching the earth in both the visible and IR regions and the reradiated IR energy are under-utilized by current technology.

Several approaches have been pursued to harvest energy from the sun. Conversion of solar energy to electricity using photovoltaic cells is the most common. An alternative to photovoltaics is the rectenna, which is a combination of a receiving antenna and a rectifier. The initial rectenna concept was demonstrated for microwave power transmission by Raytheon Company in 1964 [2]. This illustrated the ability to capture electromagnetic energy and convert it to DC power at efficiencies approaching 84\% [3]. Since then much research has been performed to extend the concept of rectennas to the infrared and visible regime for solar power conversion. Progress has been made in fabrication and characterization of metal-insulator-metal diodes for use in an infrared rectenna [45]. It has been demonstrated that optical antennas can couple electromagnetic radiation in the visible in the same way as radio antennas do at their corresponding wavelengths [6].

The major technical challenges continue to be in developing economical manufacturing methods for large-scale fabrication of antenna-based solar collectors. Further research is required to improve the efficiency of rectification of antenna induced terahertz currents to a usable DC signal. The material properties and behavior of antennas/circuits in the $\mathrm{THz}$ solar regions need to be further characterized.

\subsection{Limitations of Photovoltaic Technology}

Traditional p-n junction solar cells are the most mature of the solar energy harvesting technologies. The basic physics of energy absorption and carrier generation are a function of the materials characteristics and corresponding electrical properties (i.e. bandgap). A photon need only have greater energy than 
that of the band gap in order to excite an electron from the valence band into the conduction band. However, the solar frequency spectrum approximates a black body spectrum at $\sim 6000 \mathrm{~K}$, and as such, much of the solar radiation reaching the Earth is composed of photons with energies greater than the band gap of silicon. These higher energy photons will be absorbed by the solar cell, but the difference in energy between these photons and the silicon bandgap is converted into heat (via lattice vibrations - called phonons) rather than into usable electrical energy. For a single-junction cell this sets an upper efficiency of $\sim 20 \%$.

The current research path of implementing complex, multijunction PV designs to overcome efficiency limitations does not appear to be a cost-effective solution. Even the optimized PV materials are only operational during daylight hours and require direct (perpendicular to the surface) sunlight for optimum efficiency.

\subsection{Economical Alternative to PV}

We have developed an alternative energy harvesting approach based on nantennas that absorb the incident solar radiation. In contrast to PV, which are quantum devices and limited by material bandgaps, antennas rely on natural resonance and bandwidth of operation as a function of physical antenna geometries.

The NECs can be configured as frequency selective surfaces to efficiently absorb the entire solar spectrum. Rather than generating single electron-hole pairs as in the PV, the incoming electromagnetic field from the sun induces a time-changing current in the antenna. Efficient collection of the incident radiation is dependent upon proper design of antenna resonance and impedance matching of the antenna. Recent advances in nanotechnology have provided a pathway for large-scale fabrication of nantennas.

\section{THEORY OF OPERATION}

We have designed nantenna elements that capture electromagnetic energy from naturally occurring solar radiation and thermal earth radiation. The size of the antenna is relative to the wavelength of light we intend to harvest. The basic theory of operation is as follows: The incident electromagnetic radiation (flux) produces a standing-wave electrical current in the finite antenna array structure. Absorption of the incoming EM radiation energy occurs at the designed resonant frequency of the antenna [7].

When an antenna is excited into a resonance mode it induces a cyclic plasma movement of free electrons from the metal antenna. The electrons freely flow along the antenna generating alternating current at the same frequency as the resonance. Electromagnetic modeling illustrates the current flow is toward the antenna feedpoint. In a balanced antenna, the feedpoint is located at the point of lowest impedance. Figure 1 was acquired from modeling the electromagnetic properties of an infrared spiral antenna. The e-field is clearly concentrated at the center feedpoint. This provides a convenience point to collect energy and transport it to other circuitry for conversion.

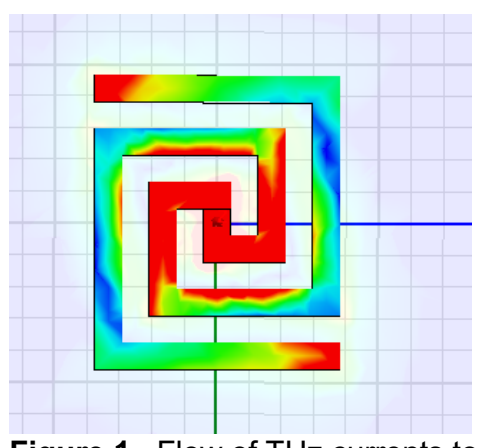

Figure 1. Flow of $\mathrm{THz}$ currents to feedpoint of antenna. Red represents highest concentrated E field. Modeled with Ansoft HFSS [8].

Antennas have electromagnetic radiation patterns, which allow them to exhibit gain and directionality and effectively collect and concentrate energy, as illustrated in Figure 2.

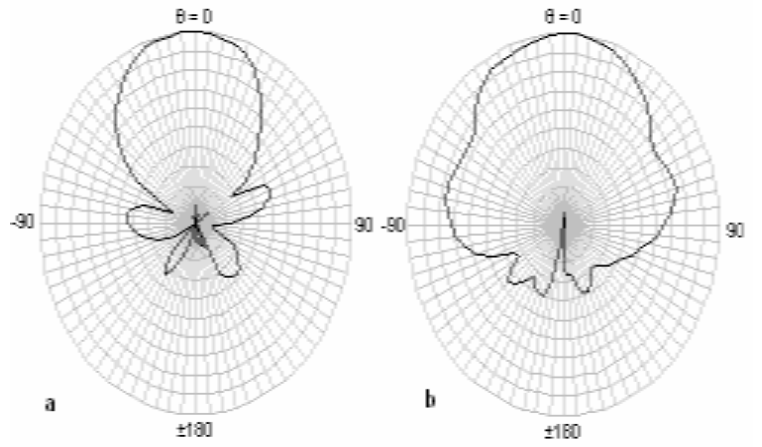

Figure 2. Typical electromagnetic radiation patterns of antenna.

The nantenna radiation pattern displays angular reception characteristics, resulting in a wider angle of incidence exposure to thermal radiation than typical PV. Any flux from the sun that falls within the radial beam pattern of the antenna is collected. This property is a critical antenna characteristic that optimizes energy collection from the sun as it moves throughout the horizon. Thus, it may be possible to reduce the need for mechanical solar tracking mechanisms. It also provides designers another mechanism to increase the efficiency of antenna arrays through the expansion of the radial field. Antennas by themselves do not provide a means of converting the collected energy. This will need to be accomplished by associated circuitry such as rectifiers. As illustrated in Fig 2, the electrical size of the antenna (comprised of radiation beam pattern) is much larger than the physical size of the antenna. The virtual large surface area antenna focuses the electromagnetic energy onto the nano-sized energy conversion material fabricated at the antenna feedpoint. Theoretical efficiency is improved by the enhanced radiation capture area of the antenna.

\section{ENERGY CONVERSION METHODS}

This research has demonstrated that infrared rays create an alternating current in the nantenna at $\mathrm{THz}$ frequencies. Commercial grade electronic components cannot operate at that switching rate without significant loss. Further research is planned to explore ways to perform high frequency rectification. This requires embedding a rectifier diode element into the antenna structure. One possible embodiment is metalinsulator-insulator-metal (MIIM) tunneling-diodes. The MIIM device consists of a thin barrier layer and two dielectric layers 2 
(oxide) sandwiched between two metal electrodes with different work functions. The device works when a large enough field causes the tunneling of electrons across the barrier layers. A difference in the work function between the metal junctions produces non-linear effects resulting in high-speed rectification. The thinner the insulated layers become the higher the non-linear effects. If one thinks about the location of an electron at a certain time being a probability curve then theoretically this concept can be thought of as the shift of the probability curve representing the electron location toward the other side of the insulated material. The thinner the insulation material thus increases this shift in probability toward the outside of the insulated material creating an increase in the nonlinear response.

The output of the rectifiers can be dc-coupled together, allowing arrays of antennas to be networked together to further increase output power capacity. This is conceptually illustrated in Figure 3.

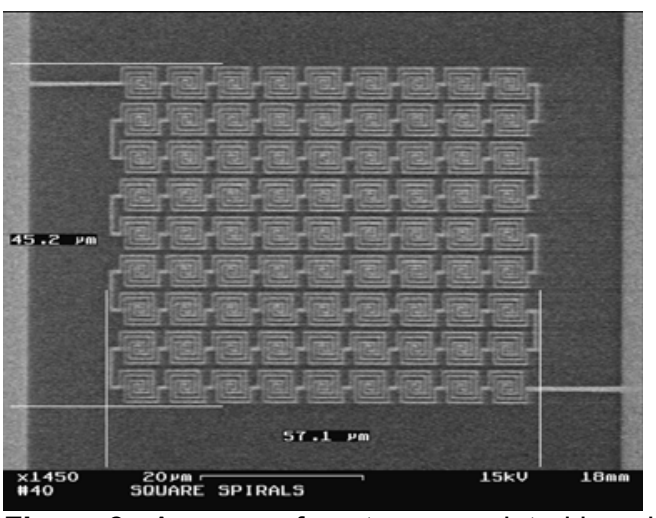

Figure 3. An array of nantennas, printed in gold and imaged with a scanning electron microscope. The deposited wire is roughly a thousand atoms thick. A flexible panel of interconnected nantennas may one day replace heavy, expensive solar panels.

\section{PROOF OF CONCEPT THROUGH MODELING}

Frequency Selective Surface (FSS) structures have been successfully designed and implemented for use in radio (RF) and microwave frequency applications. The classic laws of physics apply for adapting microwave applications to the infrared. Innovative INL research has further optimized FSS designs for operation in the Terahertz (THz) and infrared (IR) spectrums.

It is recognized that several numerical analysis techniques can be employed for electromagnetic analysis. The basis for our research and development is the adaptation of the 'Method of Moments' technique, which is a numerical computational method of solving linear partial differential equations associated with electromagnetic fields. Ohio State University has implemented a method of moments based algorithm in a software product, termed Periodic Method of Moments (PMM), which was developed for designing military RF frequency selective surfaces. The details of this code are discussed in [9]. Ohio State's PMM serves as the analysis engine for the INL 'design and modeling' methods.

\subsection{Analytical Model - RLC Circuit}

To model NEC structures it is first necessary to understand the electrical equivalent circuit and basic theory of operation. The primary antenna structure studied in the initial design of an $\mathrm{NEC}$ is a periodic array of square-loop antennas. Its RLC circuit analog is shown in Figure 4. The electrical behavior of the structure is described as follows. The metal loops give inductance to the NEC as thermally-excited radiation induces current. The gaps between the metallic loops and the gap within the loop compose capacitors with a dielectric fill. A resistance is present because the antenna is composed of lossy metallic elements on a dielectric substrate. The resulting RLC circuit has a resonance "tuned" filter behavior [7].

It is evident that the proper selection of element and substrate material is important and contributes to the RLC parameters. The electro-optical characteristics of the NEC circuit and the surrounding media have the effect of shaping and optimizing the spectral response.

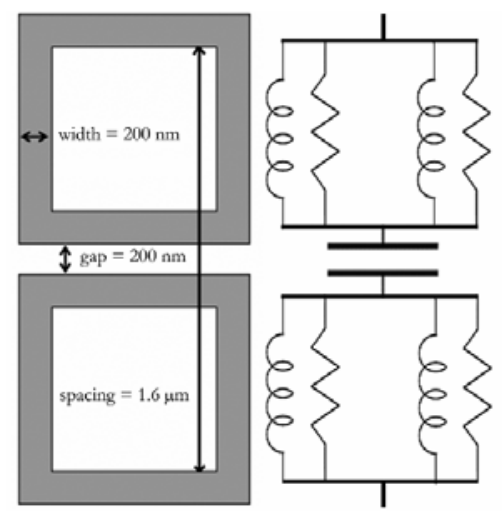

Figure 4. Square FSS element and its RLC circuit analog.

Our current designs incorporate an antenna layer, a dielectric standoff layer, and a ground plane. (see figure 5) The stand off layer serves as an optical resonance cavity. The NEC-toground plane separation acts as a transmission line that enhances resonance. The thickness of the standoff layer is selected to be a $1 / 4$ wavelength to insure proper phasing of the electromagnetic energy.

\section{Incident wave}

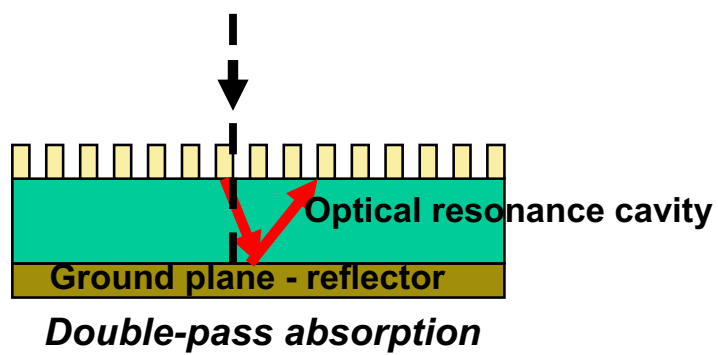

Figure 5. Side view of NEC structure showing path of incident wave.

\subsection{Frequency-Dependent Modeling}

The material properties of circuits in the infrared (IR) regime are not well characterized. It was necessary to adapt the PMM modeling software to account for the frequency-dependent optical properties of dielectric and antenna conductor materials. 
The optical functions ( $n$ and $k$ ) of various materials that comprise NEC devices were determined using spectroscopic ellipsometry. These values characterize how a material responds to excitation by light of a given wavelength. One representation is the complex index of refraction, $\widetilde{n}$, where the real part $n$ is the index and the imaginary part, $k$, is the extinction coefficient. The index, $n$, describes phase velocity of light in a material compared to propagation in a vacuum. The absorption of light is governed by the extinction coefficient, $k$. These quantities also determine the amount of light reflected and transmitted at an interface between two materials. This allows accurate simulation of antenna behavior at thermal wavelengths.

It was demonstrated that the models accurately predicted the physics of NEC energy absorption. This provides visualization of infrared thermal behavior that cannot be directly measured and supports rapid prototyping of nano-structure devices.

\section{VALIDATION OF NEC CONCEPT}

A periodic array of loop antennas was designed for resonance at 10um. This is the region of maximum thermal re-radiated emission from the earth's absorption. All required antenna geometric and material properties were entered into the PMM model. The PMM model plots the reflection and transmission spectra for the electric field and the power spectra of the NEC antenna. The PMM tool was previously validated [10] by comparing experimental and modeled datasets. However, larger datasets for specific applications are required before deriving correlation and error bounds. It is anticipated that the research team will collect future datasets for the purpose of estimating model specific applications. The following emissivity plot was acquired to estimate NEC efficiencies at the 8-12 micron wavelength supporting the reradiated heat energy application (Figure 6).

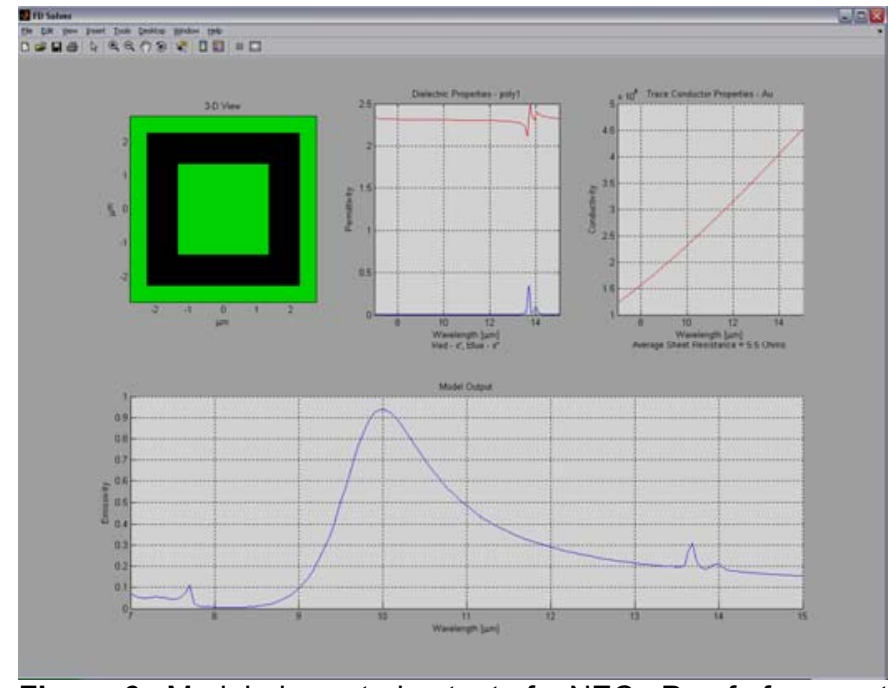

Figure 6. Modeled spectral output of a NEC. Proof of concept based on loop antenna structure, with a 10um resonance.

The output of the model is in units of emissivity versus wavelengths. It is referenced to a blackbody. A theoretical efficiency of $92 \%$ was demonstrated at a peak resonance of 10um. Efficiency is defined as the ratio of the power accepted by the antenna to the power emitted by the sun (reaching the earth's surface) over a defined frequency range. The halfpower bandwidth of the antenna is $9.2 \mathrm{um}$ to $12 \mathrm{um}$, easily covering the energy region of interest.

Additional validation modeling was performed using the Ansoft HFSS tools [8]. Proof of principle of the ability to collect and concentrate thermal energy to a focal point was modeled using a complementary square spiral antenna. The following field overlay plot (Figure 7) was acquired.

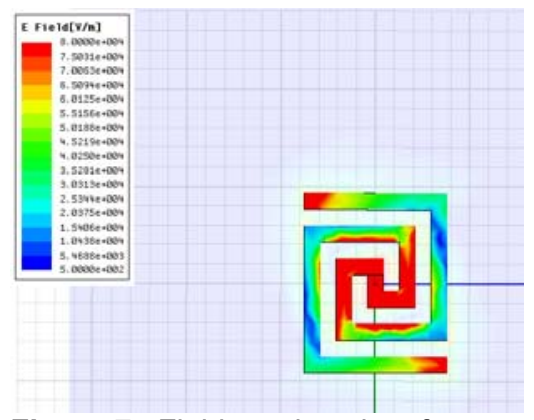

Figure 7. Field overlay plot of a square-spiral nantenna at infrared wavelengths.

A field overlay plot is representative of the E-field $[\mathrm{v} / \mathrm{m}]$ on a surface. The NEC antenna is the surface of interest. A linear frequency sweep of $1.0 \mathrm{THz}$ to $40.0 \mathrm{THz}$, at $1 \mathrm{THz}$ step size, was performed. The propagation of the e-field through the antenna structure was calculated as a function of different stimulus amplitude, frequency and phase. We further visualize how the field propagates throughout the volume of the NEC structure by animating the plot versus phase (time) when the NEC antenna is 'excited' by an incoming electromagnetic wave, representative of solar energy. It is demonstrated that surface currents flow and are concentrated into the antenna feed points.

\section{PROOF OF CONCEPT THROUGH SMALL SCALE PROTOTYPE}

\subsection{Laboratory-scale Silicon Wafer Prototypes}

The original proof-of-concept was prototyped using standard semiconductor integrated circuit fabrication techniques [11] as outlined in Figure 8. Prototype fabrication was performed at University of Central Florida under subcontract. Electronbeam lithography (EBL) was employed for fabrication of arrays of loop antenna metallic structures on dielectric and semiconductor substrates.

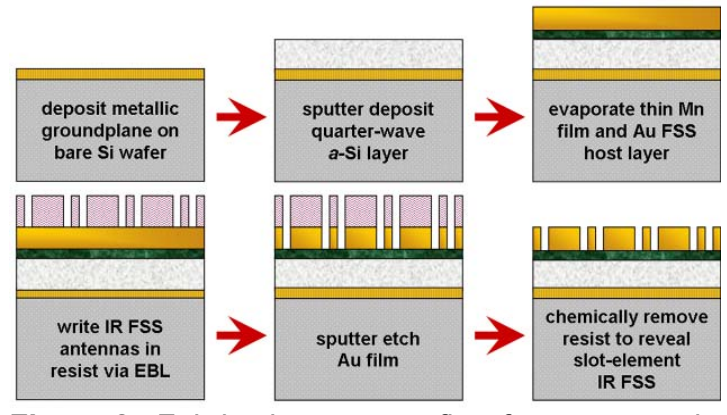

Figure 8. Fabrication process flow for a square-slot IR FSS.

E-beam lithography provided a convenient way to systematically investigate dimensional, spacing, and 
geometrical effects in a controlled manner. This approach allowed for systematic trade studies. However, the throughput and cost limitations of EBL do not support large scale manufacturing. The completed structure is shown in Figure 9.

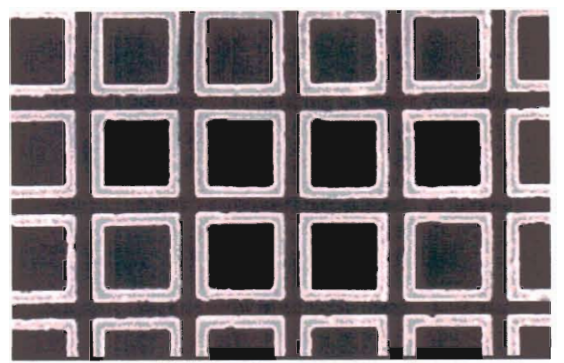

Figure 9. SEM micrograph of a small portion of the completed IR NEC. The entire IR FSS covers 40 square millimeters and is composed of $9.76 \cdot 10^{8}$ elements.

\subsection{Precursors to Roll-to-Roll Manufacturing - Nanoforming Process}

Well-established FSS theory indicates that nano-scale antennas could be fabricated for use in any region of the EM spectrum. However, feature sizes required for an NEC required the use of electron beam lithography (EBL), x-ray lithography or some other ultra-high resolution fabrication means. These techniques are generally very slow and expensive. This has limited NEC's to small sizes and has severely compromised their utility. However, due to recent advancements in ultra-high-resolution lithography and roll-to-roll (R2R) processing of flexible polymer films, low cost manufacturing is now possible.

MicroContinuum, Inc. pioneered the use of sophisticated R2R technology for rapid, low-cost production of complex multilayer film structures. A key feature of this highly innovative manufacturing technique is that a "master pattern", which can be relatively expensive, is used to mechanically "print" the precision pattern (analogous to a printing plate in the graphic arts field) on an inexpensive flexible substrate. This pattern, in turn, is used to create the metallic loop elements of the NEC device over large areas.

It is recognized that continuous large-scale production of complex multi-layer functional devices possessing nanoscale features is a monumental problem. Coupling this with a requirement for producing these devices on flexible, shape conforming substrates complicates this problem further. We have addressed these concerns by designing a multi-step rollto-roll manufacturing process for continuous fabrication of these unique optical devices. This comprises the first demonstrated large-scale manufacture of multi-layer functional nanoscale devices on flexible substrates.

\subsection{Development of a Master Pattern}

One of the most important aspects of fabricating the NEC is an understanding of the tolerances required to make structures behave like antennas at infrared wavelengths. Tolerances were derived through extensive modeling studies evaluating impacts of changing the geometric parameters including: antenna wire width/depth, antenna periodicity, gap size between adjacent antennas, etc. Many parameters are co-dependent and required use of optimization algorithms.
The resulting NEC geometry was incorporated into a master template (see Figure 10) fabricated on an 8-inch round silicon wafer. The template consists of approximately 10 billion antenna elements. The fidelity between the nantenna design and the replicated master template is excellent.

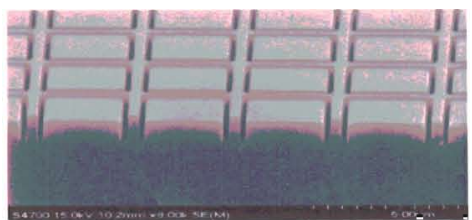

Figure 10. Electron microscope image of master template

\subsection{Prototype NEC Devices}

Processes have been developed to form nantennas onto polyethylene $(\mathrm{PE})$ in a stamp and repeat process. Prototype NEC structures have been fabricated onto flexible substrates. Using this semi-automated process, we have produced a number of 4-inch square coupons that were tiled together to form sheets of NEC structures. (See Figures $11 \mathrm{a}$ and $\mathrm{b}$ ) Prior to fully automated roll-to-roll scale up, all tooling and processes are being further developed and optimized using the semi-automated lab equipment.

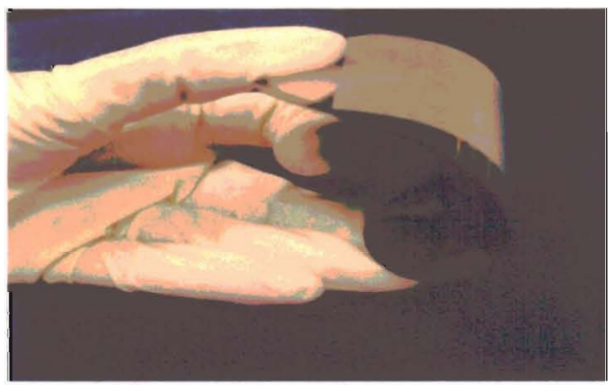

Figure 11a. Prototype FSS structure on flexible substrate.

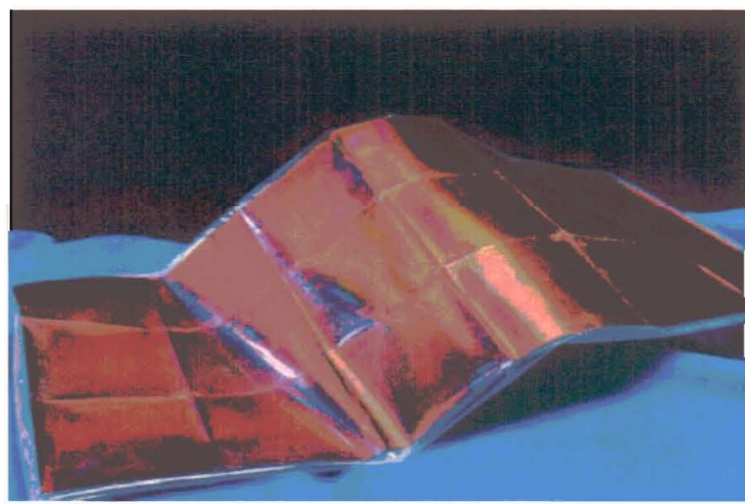

Figure 11b. Nantenna sheet, stitched together from 18 coupons.

\section{PROOF OF CONCEPT THROUGH EXPERIMENTAL MEASUREMENTS}

\subsection{Silicon Wafer Prototype}

Experimental measurements were performed on a prototype consisting of a $1.0 \mathrm{~cm}^{2}$ array of loop nantennas fabricated on a silicon substrate. This rigid substrate prototype served as the 
precursor to current work on large-scale flexible substrate nantennas.

The IR nantennas were designed to operate as a reflective bandpass filter centered at a wavelength of $6.5 \mathrm{um}$. The spectral surface characteristics from 3 to $15 \mathrm{um}$ were studied using spectral radiometer and FTIR analysis methods. The prototype was elevated to a temperature of $200^{\circ} \mathrm{C}$ and its spectral radiance spectrum was compared to blackbody emission at $200^{\circ} \mathrm{C}$. Maximum contrast is over $90 \%$ between emission near $4 \mu \mathrm{m}$ and emission at resonance [10].

A further metric of IR FSS performance is its spectral emissivity. This ratio of IR FSS radiance to blackbody radiance is shown in Figure 12. The emissivity approaches unity at resonance, indicating the IR FSS radiates like a blackbody at a specific wavelength, with a steep drop off to shorter wavelengths and a more gradual drop off in emission to longer wavelengths.

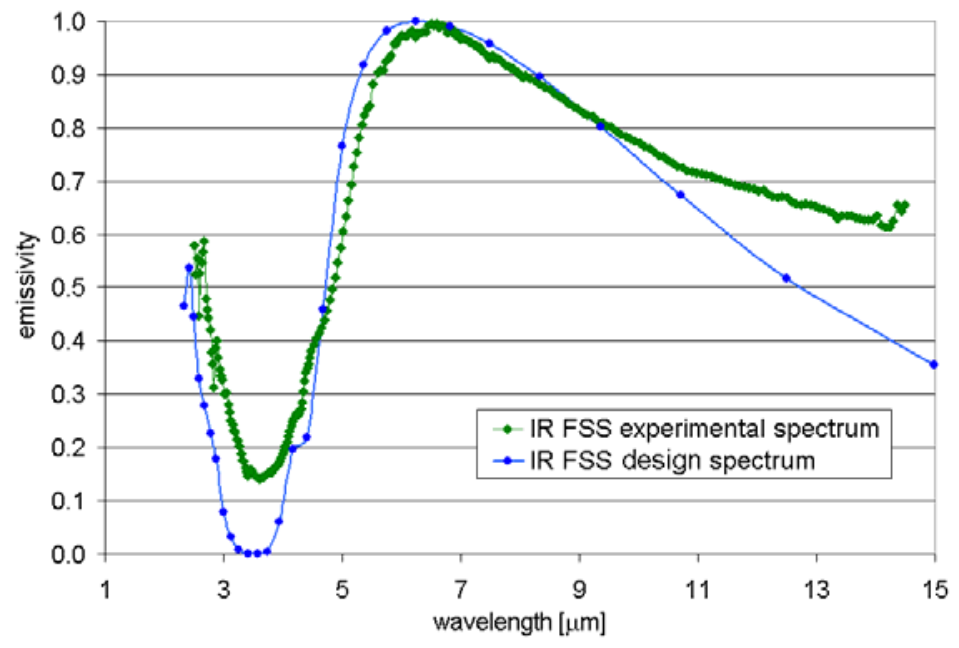

Figure 12. Demonstrated success in energy collection. Validated using a spectral radiometer and a FTIR.

The experimental spectrum closely correlates to the modeled spectrum. Peak resonance was achieved at the $6.5 \mathrm{um}$ wavelength. Resonant wavelengths are attainable by varying FSS parameters such as the standoff layer thickness and FSS element size and distribution. This basic design can be adapted to specific NEC implementations. Furthermore, out-of-band emission is $90 \%$ less than emission at resonance, making this IR FSS an excellent narrowband emissive energy concentrator or reflective filter.

\subsection{Flexible Substrate NEC Prototype}

Phase two prototypes, manufactured with manual methods using flexible polymer-based substrates, are in the process of being experimentally tested. Initial thermal characterization has been performed using high-resolution thermal cameras (Figure 13a). Initial results demonstrate proof of selective thermal energy collection.

Figure 13b shows a circular NEC structure at the center of a metal plate. When excited by thermal energy the metal reference plate and the NEC structure have distinct emissivity contrasts, per the modeled design. This behavior will be further optimized to increase the collection efficiency of thermal radiation in the $10 \mathrm{um}$ solar spectrum.

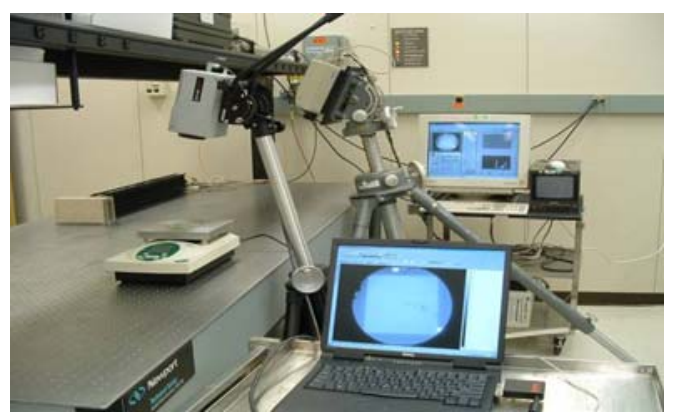

Figure 13a. Experimental setup for thermal characterization of prototypes.

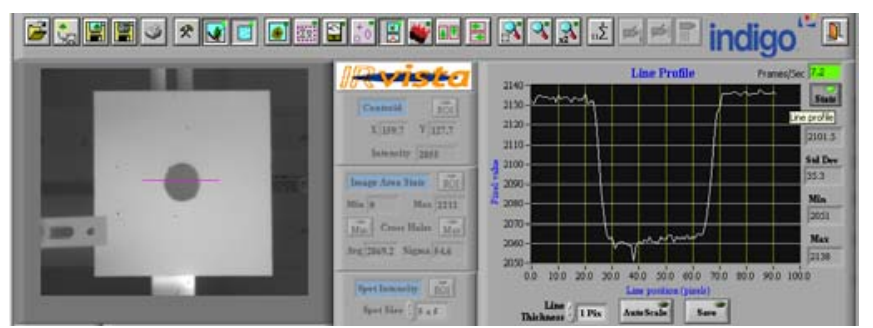

Figure 13b. Optical and graphical experimental results from thermal characterization.

\section{APPLICATIONS}

Applications for this technology are very diverse. It is conceivable that nantenna collectors, combined with appropriate rectifying elements, could be integrated into the 'skin' of consumer electronic devices to continuously charge their batteries. Economical large-scale fabrication would support applications, such as, coating the roofs of buildings and supplementing the power grid. Due to the ability of integrating the nanostructures into poly materials it is possible that they could even be directly fabricated into polyester fabric.

The NEC devices can be optimized for collection of discrete bands of electromagnetic energy. Double-sided panels could absorb a broad spectrum of energy from the sun during the day, while the other side might be designed to take in the narrow frequency of energy produced from the earth's radiated heat or potentially residual heat from electronic devices. Available flux that reaches the earth's surface in the .8-.9 um range is about $800 \mathrm{w} / \mathrm{m}^{2}$ at its zenith. While visible light flux is dependent on cloud cover and humidity, incident light in this range is a constant during daylight hours.

This technology may also support several emerging applications, including passive energy management products, such as building insulation, window coatings, and heat dissipation in small electronic consumer products, such as, computers. The nantennas are broadband collectors of energy with a tailorable spectral emission response. This in effect generates a frequency selective distribution of energy. This potentially will collect unwanted energy (residual or incident heat) and redistribute it at other innocuous wavelengths. 


\section{CONCLUSIONS AND FUTURE WORK}

Both modeling and experimental measurements demonstrate that the individual nantennas can absorb close to 90 percent of the available in-band energy. Optimization techniques, such as, increasing the radial field size could potentially increase this efficiency to even higher percentages. More extensive research needs to be performed on energy conversion methods to derive overall system electricity generation efficiency. The circuits can be made from any of a number of different conducting metals. The nantennas can be formed on thin, flexible materials like polyethylene.

Further laboratory evaluations of the flexible substrate NEC prototypes are planned. Manufacturing methods will continue to be refined to support roll-to-roll manufacturing of the nanostructures. Future work will focus on designing the nantenna structure for operation in other wavelengths. By further shaping the spectral emission of the NEC it may be possible to concurrently collect energy in the visible, nearinfrared and mid-infrared regions.

This research is at an intermediate stage and may take years to bring to fruition and into the market. The advances made by our research team have shown that some of the early barriers of this alternative PV concept have been crossed and this concept has the potential to be a disruptive and enabling technology. We encourage the scientific community to consider this technology along with others when contemplating efforts and resources for solar energy.

\section{ACKNOWLEDGMENTS}

The authors would like to thank Glenn Boreman and staff at the CREOL, University of Central Florida, for assistance with initial prototyping of FSS structures and radiometric measurements. We also recognize the support of Ben Munk and Ron Marhefka of Ohio State University for their support in electromagnetic modeling. The authors would like to thank Judy Partin for her contributions in thermal characterization and analysis. The authors extend a special thank you to Jay Rsykamp at NAVSEA for his support and much needed direction. This work was supported by the U.S. Department of Energy, Office of Nuclear Energy, under DOE Idaho Operations Office Contract DE-AC07-05ID14517

\section{References}

[1] Guy J. Consolmagno and Martha W. Schaefer, World's Apart: A Textbook in Planetary Sciences

(1994) Englewood Cliffs, NJ: Prentice Hall.

[2]http://www.mtt.org/awards/WCB's\%20distinguished\%20car eer.htm

[3] http://www.kurasc.kyoto-u.ac.jp/plasma-group/sps/history2e.html

[4] I. Wilke, Y. Oppliger, W. Herrmann, F.K. Kneubuhl: Appl. Phys. A58, 329-341 (1994)

[5] Subramanian Krishnan, Shekhar Bhansali, Kenneth Buckle, and Elias Stefanakos, "Fabrication and Characterization of Thin-film Metal-Insulator-Metal Diode for use in Rectenna as Infrared Detector”, Mater. Res. Soc. Symp.Proc. Vol 935.

[6] Alda, J. Rico-García, J. López-Alonso,and G. Boreman, "Optical antennas for nano-photonic applications," Nanotechnology, vol. 16, pp. S230-4, 2005
[7] B. A. Munk, "Frequency Selective Surfaces: Theory and Design". New

York: Wiley, 2000, pp. 2-23.

[8]Ansoft High Frequency Structure Simulator v10 User's Guide, Ansoft Corporation, (2005)

[9] L. W. Henderson, "Introduction to PMM, Version 4.0," The Ohio State Univ., EletroScience Lab., Columbus, OH, Tech. Rep. 725 347-1, Contract SC-SP18-91-0001, Jul. 1993.

[10] B. Monacelli, J. Pryor, B. Munk, D. Kotter, G. Boreman, "Infrared Frequency Selective Surfaces based on circuit-analog square loop design". IEEE Transactions on antennas, Vol. 53, No.2 Feb 2005

[11] B. Monacelli, J. Pryor, B. Munk, D. Kotter, G. Boreman, "Infrared Frequency Selective Surfaces:Square loop versus Square-Slot Element Comparison” AP0508-0657, Aug 2005 